\title{
Timetabling Problems at the TU Eindhoven
}

\author{
John van den Broek, Cor Hurkens, and Gerhard Woeginger \\ Department of Mathematics and Computer Science, \\ Eindhoven University of Technology, \\ Den Dolech 2, 5600 MB Eindhoven, The Netherlands \\ $j \cdot j \cdot j \cdot v \cdot d . b r o e k @ t u e . n l$, wscor,gwoegi\}@win.tue.nl
}

\begin{abstract}
The students of the Industrial Design department at the TU Eindhoven are allowed to design part of their curriculum by selecting courses from a huge course pool. They do this by handing in ordered preference lists with their favorite courses for the forthcoming time period. Based on this information (and on many other constraints), the department then assigns courses to students. Until recently, the assignment was computed by human schedulers who used a quite straightforward greedy approach. In 2005, however, the number of students increased substantially, and as a consequence the greedy approach no longer yielded acceptable results.

This paper discusses the solution of this real-world timetabling problem. We present a complete mathematical formulation of it, and we explain all the constraints resulting from the situation in Eindhoven. We solve the problem using lexicographical optimization with four subproblems. For all four subproblems, an elegant integer linear programming model is given which easily can be put into CPLEX. Finally, we report on our computational experiments and results around the Eindhoven real-world data.
\end{abstract}

\section{Introduction}

In February 2005, outraged students of the Industrial Design department were protesting at the TU Eindhoven (The Netherlands). Uproar and revolt were in the air. What had happened? Here is the story. The academic year of the roughly 350 students of Industrial Design is split into a number of periods. In every period, every student must do a number of small courses. There is a pool of roughly 55 courses to choose from, and every student submits an ordered preference list with his/her 10 favorite courses to the department. Based on all the ordered preference lists, a scheduler at the department then assigns roughly four courses to every student. Until recently, the scheduler was a human decisionmaker who essentially applied a hand-woven greedy assignment procedure.

In February 2005, the students were absolutely dissatisfied with the work of the human scheduler: many of them did not get the courses which they would have liked to get; many of them were assigned to courses which they really did not want to do; and more than 150 out of the 350 students received courses that were not listed on their preference list! 
The department of Industrial Design realized that they had a problem. They also realized that they did not know how to solve this problem. The number of students had increased substantially, and the timetabling problem had become much larger, much harder, and much more complex. Hence, the department contacted the local experts on the campus: us. They were hoping to find a somewhat better assignment through computer programs. They explained their problem to us (in a certain problem formulation No. 1), and we happily told them that we would be able to solve it: the problem (in formulation No. 1) could be modeled as a network flow problem, and hence is solvable in polynomial time. Unfortunately, it turned out that formulation No. 1 was not a complete formulation of the problem: they had forgotten to inform us about a number of additional restrictions that lead to a new, more difficult assignment problem (in formulation No. 2), which eventually turned out to be NP-hard.

This paper is a report on the course assignment problem of the Industrial Design department. We describe the assignment problem in its (incomplete) formulation No. 1 and in its (complete) formulation No. 2. We show that formulation No. 1 yields a tractable problem, whereas formulation No. 2 yields an intractable problem. Our main contribution is a careful case study of the complete problem formulation. We design an elegant integer linear programming model for it, with roughly 9000 variables and roughly 7000 constraints. Putting this ILP model into CPLEX yields excellent results within moderate computation times. We describe the ILP model in detail, and we report on our computational experiments with the real-world data of the Industrial Design department.

Structure of the paper. The rest of the paper is structured in the following way. In Section 2 we give a literature review of university and school timetabling. Section 3 contains a detailed description of the problem we solved for the department of Industrial Design. The problem is formulated as an integer linear program which is described in Section 4 Section 5 contains the computational results. Some conclusions are given in Section 6 .

\section{Literature Review}

The literature contains a large number of variants of the timetabling problem. These variants differ from each other by the type of institution involved (university or high school) and by the type of constraints. The annotated bibliography of timetable construction by Schmidt and Ströhlein 22 lists many papers that appeared before 1980. Schaerf [21] gives a survey of the various formulations of timetabling problems and classifies the timetabling problem into the following three main classes: school timetabling, examination timetabling and course timetabling. Of course this classification is crude, and there are many real-world timetabling problems that fall in between two of these classes. For surveys of timetabling methods and applications see de Werra [11, Burke et al. [3], Carter and Laporte [8] and Burke and Petrovic [5]. 
The basic school timetabling problem is also known as the class-teacher model. The simplest problem consists in assigning lectures to periods in such a way that no teacher or class is involved in more than one lecture at a time. Even et al. 13. proved that there always exists a solution of this simplest version of the school timetabling problem, unless a teacher or class is involved in more lectures than there are time slots. Alternative formulations of the school timetabling problem with more constraints can be found for example in Even et al. [13], Garey and Johnson [15] and de Werra [11. Daskalaki and Birbas [10] provide an integer programming formulation of the class-teacher problem and solve it with a twostage relaxation procedure. The problem is formulated as a set packing problem with side constraints by Avella and Vasilev [2. They tighten the formulation by adding the valid inequalities of the Set Packing polytope and introduce some new valid inequalities.

University timetabling can be classified into two categories: course and examination timetabling. Petrovic and Burke [19] discuss problem statements and give an overview on recent research results on university timetabling. The main differences between course timetabling and examination timetabling are that examination timetabling has only one exam for each course, that the time conflict condition is strict, and that several exams can be done simultaneously in one room. Examples for additional soft constraints are: students can do at most one exam per day, and students may not have too many consecutive exams. Schaerf 21 gives an integer linear programming formulation of the examination timetabling problem and describes some alternative variants of the problem. Carter and Laporte [7] provide an overview on examination timetabling.

The course timetabling problem consists in scheduling a set of lectures for each course within a given number of rooms and time period. The main difference from the school timetabling problem is that university courses can have common students, whereas school classes are disjoint sets of students. De Werra 11] gives a binary integer programming formulation. An overview on course timetabling problems is given by Carter and Laporte 8 and Schaerf 21 discusses some of the most common variants of the basic formulation. The design and implementation of a decision support system for constructing a combined university course-examination timetable is reported by Dimopoulou and Miliotis [12]. They also take into account the increased flexibility of students' preferences for specific classes.

One variant is called the grouping subproblem or student scheduling problem. If the number of students is too large for one room, courses are split into groups of students and there are conditions on the minimum and maximum number of students that can be assigned to each group. A student is required to take a certain number of courses, which they have to select themselves after a timetable is made available. The problem consists of assigning a student to a specific group of a course for a given fixed timetable such that students are satisfied and there are no time conflicts, see Busam 6], Feldman and Golumbic [14 and Laporte and Desrochers [16]. 
Cheng et al. 9] discuss the Student Scheduling Problem (SSP) as it generally applies to high schools in North America. They define the problem as the assignation of courses and a specific section to each student. The objective is to fulfill student requests, providing a conflict-free schedule. They show that the problem is NP-hard and discuss various multi-commodity flow formulations with fractional capacities and integral gains. The main difference between the SSP and our timetabling problem is that for the SSP all courses on the preference list of the students have to be assigned to students. This results with most practical cases in an empty feasible solution set.

Laporte and Desrochers 16] give a mathematical formulation of the student scheduling problem. They formulate the problem as an optimization problem splitting the requirements into hard and soft ones. The only hard constraint in their model is that student course selections must be respected. Time conflicts for students are soft constraints. When time conflicts occur students are advised to make a different course selection. The problem is then solved in three phases: in the first one the algorithm searches for an admissible solution, in the second section enrollments are balanced and in the third the room capacities have to be respected. Tripathy 23 formulated the student scheduling problem as an integer linear programming problem and uses Lagrangian Relaxation to solve it. Sabin and Winter 20] use a greedy approach that is moderated by an intelligent ordering of the students. Miyaji et al. 18 apply goal programming.

McCollum 17. explains that for university timetabling there is still a gap between a successful research project and what is needed in practice. He tries to bridge this gap between research and practice by providing up-to-date information from practice which is needed by researchers. Burke et al. 4 and Zampieri and Schaerf 24] note that many of the search methodologies described in the literature are not applicable in most educational institutions, because they are simplified too much.

Carter and Laporte [8] note that they were 'somewhat surprised to discover that there are very few course timetabling papers that actually report that the (research) methods have been implemented and used in institution'. McCollum 17. explains that the situation has hardly changed in the last decade. Our paper is an example of a successful implementation of a mathematical programming model for a specific course timetabling problem.

\section{Problem Description}

At our first meeting, the Industrial Design department explained the problem to us in a certain problem formulation No. 1; see Section 3.1. This problem can be modeled as a network flow problem, and hence is solvable in polynomial time; see Ahuja et al. [1.

Unfortunately, we learnt after some time that formulation No. 1 was not a complete formulation of the problem. They actually had forgotten to tell us about a number of additional restrictions that lead us to a new, more difficult assignment problem formulation No. 2. Section 3.2 describes formulation No. 2 . 
Table 1. Example of preference lists

\begin{tabular}{ccccccc}
\hline Student & $r_{s}$ & P1 & P2 & P3 & $\ldots$ & P10 \\
\hline s040202 & 4 & DAC03 & DA247 & DA125 & $\ldots$ & DA405 \\
s040203 & 4 & DA619 & DA125 & DA201 & $\ldots$ & DA616 \\
s040204 & 4 & DA418 & DA242 & DA402 & $\ldots$ & DA621 \\
\hline
\end{tabular}

\subsection{Problem Formulation No. 1}

At the first meeting with the Industrial Design department, we were told that every student hands in a preference list of at most 10 courses and requests a certain number of courses. The only constraints are that a student cannot do two courses at the same time and there is a maximum number of students that can be assigned to a course. This section contains a more detailed description of problem formulation No. 1 .

A set $C$ of courses and for each course $c$ an upper bound $C_{c}^{\max }$ on the number of students is given. This number depends on the preference of the teacher and the room capacity in which the course is given. Each course has one weekly meeting time which is already fixed. This weekly meeting time always consists of two consecutive hours. Two such consecutive hours are defined as one time slot. The weekly meeting time of a course is chosen from a set $T$ of disjoint time slots. $T(c)$ is defined as the time slot which is the weekly meeting time of course $c$. Hence, one of the constraints in the model is that courses $c_{i}$ and $c_{j}$ cannot be assigned to one student if $T\left(c_{i}\right)=T\left(c_{j}\right)$.

We define $S$ as the set of students. For each student $s$ the requested number $r_{s}$ of courses is given. $P_{s}$ is defined as the set of positions on the preference list for which student $s$ filled in a course. Most students have $P_{s}=\{1, \ldots, 10\}$. There are also students that hand in a smaller preference list. For instance, a student almost finishing his bachelor degree and with only one course left to do, which has to be a math course, hands in a preference list with only math courses. For a student $s$ with only six courses on its preference list we have $P_{s}=\{1, \ldots, 6\}$. Table 1 gives a few examples of preference lists. Column $P i$ gives the encoded course name of the course on position $i$ of the preference list. The parameter $c_{s p}$ is introduced and is equal to $c$ if course $c$ is on position $p$ of the preference list of student $s$.

In summary, the input of problem formulation No. 1 consists of

- a set $T$ of time slots;

- a set $C$ of courses; for every course $c \in C$ a time slot $T(c)$ and a maximum number $C_{c}^{\max }$ of participating students is given;

- a set $S$ of students; for every student $s \in S$ a set $P_{s}$ of filled positions of the preference list, a course $c_{s p}$ for each position $p \in P_{s}$ and a requested number $r_{s}$ of courses is given.

The goal is to assign as many courses to students as possible, while 
- the number of courses assigned to student $s$ does not exceed the requested number $r_{s}$,

- courses assigned to a student are on his preference list,

- courses assigned to a student do not conflict in time,

- no course exceeds its maximum number of assigned students.

This problem can be modeled as a network flow problem. A description of this network flow model is given in Appendix $\mathrm{A}$.

\subsection{Problem Formulation No. 2}

When we received the first data set from the Industrial Design department, we were very surprised: there suddenly were also lower bounds $C_{c}^{\min }$ on the number of students participating in course $c$. This yields the new constraint that a course either will not be given at all, or otherwise has at least $C_{c}^{\min }$ participating students. This new constraint cannot be modeled as a flow-constraint, and hence the maximum flow model in Appendix $\mathrm{A}$ becomes obsolete. In fact, the new constraint makes the problem NP-hard; see Appendix B. After looking at the data more carefully and after conversations with the Industrial Design department we noticed there were a lot more restrictions. This section explains these extra restrictions and defines the problem in more detail.

An academic year is divided into a certain number of teaching periods. For instance, the academic year 2005-06 is divided into six teaching periods. We define such a teaching period as a block. The Industrial Design department wants us to schedule two consecutive blocks simultaneously. Therefore, set $B$ is introduced as the set of blocks that have to be scheduled simultaneously.

In problem formulation No. 1 we assumed the workload of all courses was equal. However, there are courses with a workload of 40 hours and courses with a workload of 80 hours. In the remainder of this paper a workload of 1 corresponds with a workload of 40 hours. In Appendix B we prove that having courses with a workload 1 and courses with a workload 2 makes the problem NP-hard. For each course $c \in C$ and block $b \in B$ the parameter $w(c, b)$ is defined as the workload of course $c$ in block $b$. Hence for a course $c$ with a workload of 80 hours in block $b$ we have $w(c, b)=2$.

In problem formulation No. 2 the definition of $r_{s}$ is adjusted into the requested workload of student $s$ for $|B|$ blocks together. For every student $s$, a maximum requested workload $r_{s b}$ for each block $b \in B$ is given separately, because the requested workload of a student is not always equally divided over all blocks $b \in B$. For instance, if student $s$ has to do a practical training in block $b_{2}$ he has $r_{s}=2, r_{s b_{1}}=2$ and $r_{s b_{2}}=0$.

It was assumed in problem formulation No. 1 that a course has one meeting every week, hence it has one time slot. But there are also courses which have two weekly meetings, and hence two time slots. If courses with two time slots are introduced into problem formulation No. 1, the problem cannot be modeled as a network flow problem. 
Table 2. Examples of courses

\begin{tabular}{lccccrr}
\hline Course & Section & Time slots of meetings & wlb1 wlb2 & Min & Max \\
\hline DA242 & DAG242-1 & B1TM2, B1TA1 & 1 & 0 & 0 & 30 \\
& DAG242-2 & B1TM2, B1TA2 & 1 & 0 & 0 & 30 \\
& DAG242-3 & B1TM2, B1WA1 & 1 & 0 & 0 & 30 \\
& DAG242-4 & B1TM2, B1WA1 & 1 & 0 & 0 & 30 \\
& DAG242-5 & B1TM2, B1WA2 & 1 & 0 & 0 & 30 \\
DA247 & DAG247-1 & B1WA2, B2WA2 & 1 & 1 & 5 & 15 \\
& DAG247-2 & B1WA2, B2WA2 & 1 & 1 & 5 & 15 \\
\hline
\end{tabular}

The set $C$ of courses contains courses with multiple sections, meaning that the course is repeated during the week. Table 2 contains course DA242 as an example. For example, time slot B1TM2 stands for the second part of Tuesday morning in block 1 . The workloads of a course in block 1 and 2 are denoted by $w l b 1$ and $w l b 2$. The course DA242 has five sections which all have two time slots.

We define $I$ as the set of sections offered to the students. For every section $i \in I$ its course $c(i) \in C$ is given, a minimum number $C_{i}^{\text {min }}$ and a maximum number $C_{i}^{\max }$ of students. The meeting times for each section $i \in I$ are given as the set of time slots $T(i) \subseteq T$. There are a few courses, for example literature studies, which are not assigned to a time slot and thus $T(i)=\emptyset$.

Another constraint arises if students have specific needs, for instance when they almost finish their studies and only have one course left to pass. Then a course on the preference list of the student can be set to urgent. As long as the maximum number of students (all with an urgency) is not assigned to this course, the course has to be assigned to the student. A course which is urgent for one student has to be given. In this case, it doesn't matter whether the minimum number of students is reached or not. We define $U$ as the set containing all combinations $(s, p)$ for which course $c_{s p}$ is urgent for student $s$.

A few courses have meeting times which are spread over two blocks. See for example course DA247 in Table 2. This course has two sections and a total workload of two which is equally spread over the two blocks. If a student is assigned to a section of this course in one block he needs to be assigned to the same section of this course in the next block. Hence, it is also possible that courses are given in two blocks which are not scheduled simultaneously. If this occurs, this implies there are students already preassigned to sections if the schedule of the second block is made. Therefore, we introduce the set $F$ of fixations which contains combinations $(s, p, i)$ for which section $i$ of course $c_{s p}$ is already assigned to student $s$. This results in hard constraints that do not lead to an infeasible solution, because these students are assigned in the scheduling period before.

In summary, the input of problem formulation No. 2 consists of

- a set $B$ of blocks that have to be scheduled simultaneously;

- a set $T$ of time slots;

- a set $C$ of courses; for every course $c$ its workload $w(c, b)$ for each block $b$ is given; 
- a set $S$ of students; for every student $s$ a total requested workload $r_{s}$, a requested workload $r_{s b}$ for each block separately, a set $P_{s}$ of filled positions on the preference list and for each position $p \in P_{s}$ a course $c_{s p}$ is given;

- a set $I$ of sections; for every section $i$ its course $c(i)$, a minimum $C_{i}^{\text {min }}$ and maximum $C_{i}^{\max }$ number of students and a set of time slots $T(i) \subseteq T$ is given;

- a set $U$ of combinations $(s, p)$ for which course $c_{s p}$ is urgent for student $s$;

- a set $F$ of combinations $(s, p, i)$ for which section $i$ of course $c_{s p}$ is already preassigned to student $s$.

Our main goal is to assign workload to students as much as possible, while

- maintaining the number of students in a section below a maximum size prescribed,

- the total workload assigned to student $s$ is less than or equal to $r_{s}$,

- the workload assigned to student $s$ in block $b$ is less than or equal to $r_{s b}$,

- sections assigned to a student do not conflict in time,

- students are only assigned to a section of a course on their preference list,

- students are only assigned to one section of a course,

- student $s$ is assigned to section $i$ if $(s, p, i) \in F$.

Soft constraints are, for example, spreading students over sections, a section needing to be assigned to at least a certain minimum number of students and student $s$ having to be assigned to course $c_{s p}$ if $(s, p) \in U$.

\section{The Integer Linear Programming Model}

The problem is formulated and solved as a lexicographic optimization problem. Lexicographic optimization is a form of multi-criteria optimization in which the various objectives $f_{i}, i=1, \ldots, m$ cannot be quantitatively traded off between each other. If a solution $x$ minimizes $f_{1}$, then a solution $x^{\prime}$ minimizes $f_{2}$ if the condition $f_{1}(x)=f_{1}\left(x^{\prime}\right)$ is satisfied. In general, $x^{*}$ minimizes $f_{i}$ under the constraining conditions that $f_{1}\left(x^{*}\right)=f_{1}\left(x_{1}\right), \ldots, f_{i-1}\left(x^{*}\right)=f_{i-1}\left(x_{i-1}\right)$ where $x_{j}$ minimizes $f_{1}, \ldots, f_{j}$ for $1 \leq j \leq i-1$.

The timetabling problem is split into four subproblems which are formulated as an integer linear programming problem. The goals of the four subproblems are:

1. Maximize the number of assigned courses with an urgency.

2. Minimize the shortage of students to reach the minimum number of students of a section. Because of urgencies, some sections must be taught, but do not have enough students with this course on their preference list. We assign as many students as possible to those sections.

3. Maximize the total assigned workload. We try to assign a workload $r_{s}$ to every student $s$.

4. 'Optimize' the timetable. For example by assigning courses to students which rank high on their preference list. 
All parameters are already introduced in Section 3, Left to define are the decision variables. These are defined as follows:

$$
\begin{aligned}
& x_{s p}:=\left\{\begin{array}{l}
1 \text { if course } c_{s p} \text { is assigned to student } s \\
0 \text { otherwise }
\end{array}\right. \\
& y_{i}:=\left\{\begin{array}{l}
1 \text { if section } i \text { is assigned to one or more students } \\
0 \text { otherwise }
\end{array}\right. \\
& z_{s p i}:=\left\{\begin{array}{l}
1 \text { if section } i \text { of course } c_{s p} \text { is assigned to student } s \\
0 \text { otherwise }
\end{array}\right.
\end{aligned}
$$

The following constraints have to be fulfilled in all four subproblems:

$$
\begin{array}{rlrl}
\sum_{i \in I \mid c_{s p}=c(i)} z_{s p i} & =x_{s p} & \forall s \in S, \forall p \in P_{s} \\
\sum_{p \in P_{s}} \sum_{i \in I \mid c_{s p}=c(i)} w\left(c_{s p}, b\right) z_{s p i} & \leq r_{s b} & \forall s \in S, \forall b \in B \\
\sum_{p \in P_{s}} \sum_{i \in I \mid c_{s p}=c(i)} w\left(c_{s p}, b\right) z_{s p i} & \leq r_{s} & \\
\sum_{s \in S} \sum_{p \in P_{s}} \sum_{i \in I \mid c_{s p}=c(i), t \in T(i)} z_{s p i} \leq C_{i}^{\max } y_{i} & \forall s \in S \\
z_{s p i} & \leq 1 \\
z_{s p i} & \forall i \in I \\
x_{s p} & \in\{0,1\} \\
y_{i} \in\{0,1\} & \forall s \in S, \forall t \in T \\
z_{s p i} \in\{0,1\} & \forall i \in I \mid(s, p, i) \in F
\end{array}
$$

Constraint (1) ensures that at most one section of a course is assigned to a student. The workload assigned to a student has to be less than or equal to the requested workload of each block separately and all blocks together. This is fulfilled by constraints (2) and (3). Constraint (4) enforces that the maximum number of students for a section is not exceeded and constraint (5) ensures that at each time slot only one section is assigned to each student. If $(s, p, i) \in F$ then section $i$ of course $c_{s p}$ has to be assigned to student $s$, which is fulfilled by constraint (6).

The goal of the first subproblem is to maximize the number of assigned courses with an urgency. The constraint that a section needs to have more than a minimum number of students is not a restriction in this subproblem, because at least 
one section of a course must be given if there is a student with an urgency for this course. This first subproblem can be solved with the following ILP formulation:

$$
\begin{gathered}
U^{\max }=\max \sum_{(s, p) \in U} x_{s p} \\
(x, y, z) \text { satisfy }(1)-(9) .
\end{gathered}
$$

The next step is to minimize the shortage of students to reach the minimum number of students of a section, keeping the maximum number of assigned courses with an urgency equal to $U^{\max }$. There are sections that have to be given because they are assigned to students with an urgency for the corresponding course. Those sections are assigned to other students such that the minimum number of students for those sections is reached. The decision variable $s_{i}$ is defined as the shortage of students for section $i$. This variable gets a value larger than zero if it is not possible to assign section $i$ to the minimum number $C_{i}^{\min }$ of students. The second subproblem minimizes the total shortage $S^{\text {min }}$ of students. This results into the following ILP formulation:

$$
\begin{array}{ccc}
\min \sum_{i \in I} s_{i} & =S^{\min } & \\
\sum_{(s, p) \in U} x_{s p} & =U^{\max } & \\
\sum_{s \in S} \sum_{p \in P_{s}, c_{s p}=c(i)} z_{s p i}+s_{i} & \geq C_{i}^{\min } y_{i} & \forall i \in I \\
s_{i} & \in \mathbb{Z}_{+}, & \\
(x, y, z) \text { satisfy }(1)-(9) . &
\end{array}
$$

The third subproblem maximizes the total workload assigned to students with the restrictions that $U^{\max }$ and $S^{\text {min }}$ keep their optimal values. This maximum workload is denoted by $W^{\max }$ and is determined by the following model:

$$
\begin{array}{rlrl}
\max \sum_{s \in S} \sum_{p \in P_{s}} \sum_{b \in B} w\left(c_{s p}, b\right) x_{s p} & =W^{\max } & \\
\sum_{i \in I} s_{i} & =S^{\min } & \\
\sum_{(s, p) \in U} x_{s p} & =U^{\max } & \\
\sum_{s \in S} \sum_{p \in P_{s}, c_{s p}=c(i)} z_{s p i}+s_{i} \geq C_{i}^{\min } y_{i}, & \forall i \in I \\
s_{i} & \in \mathbb{Z}_{+}, & \forall i \in I
\end{array}
$$




$$
(x, y, z) \text { satisfy }(1)-(9) \text {. }
$$

To 'optimize' the final timetable we assign courses as high as possible on the preference lists, spread the students as equally as possible over the sections of a course and discourage the possibility that one student gets a lot of courses which are on the bottom of his preference list. Therefore, the fourth subproblem is solved. The objective function is separated into three terms and has to be minimized under the restrictions that $U^{\max }, S^{\min }$ and $W^{\max }$ keep their optimal values.

The term in the objective function to assign courses as high as possible on the preference lists is $W_{p} \sum_{s \in S} \sum_{p \in P_{s}} \sum_{b \in B} w\left(c_{s p}, b\right)\left(82-(10-p)^{2}\right) x_{s p}$. Assigning a course on top of a preference list, $p=1$ for this course, adds a lot less to the objective function than assigning a course on the bottom of the list, $p=10$ for this course. $W_{p}$ is a weighting factor and also the workload is taken into account.

If a course has multiple sections, students have to be spread as equally as possible over the sections. Therefore, $I_{c}^{\max }$ is introduced as the number of students assigned to the section of course $c$ with the most students assigned. Also the spread $S_{c}$ of course $c$ is introduced and is equal to the sum over all sections of the difference between $I_{c}^{\max }$ and the assigned number of students in each section. $S_{c}$ is added to the objective function with a weighting factor $W_{s}$.

We also discourage the possibility that one student gets a lot of courses from the 7 th to 10 th position of his preference list. A constraint is added to the model that checks whether a student gets more than one course from these positions. If so, then a penalty $W_{e}$ is paid for each 'extra' course from these positions. Therefore, the decision variable $E_{s}$ is introduced for every student $s$. This variable is equal to the 'extra' number of courses assigned to student $s$ which are from the 7 th to 10 th position of his preference list.

This results in the final ILP formulation:

$$
\begin{array}{cc}
\min W_{p} \sum_{s \in S} \sum_{p \in P_{s}} \sum_{b \in B} w\left(c_{s p}, b\right)\left(82-(10-p)^{2}\right) x_{s p}+W_{s} \sum_{c \in C} S_{c} & +W_{e} \sum_{s \in S} E_{s} \\
\sum_{s \in S} \sum_{i \in I \mid c=c(i)}\left(I_{c}^{\max }-\sum_{s \in S} \sum_{p \in P_{s}, c_{s p}=c(i)} z_{s p i} \leq I_{c(i)}^{\max } z_{s p i}\right)=S_{c} & \forall i \in I \\
\sum_{p=7}^{10} x_{s p} \leq 1+E_{s} & \forall c \in C
\end{array}
$$

$$
\begin{aligned}
\sum_{s \in S} \sum_{p \in P_{s}} \sum_{b \in B} w\left(c_{s p}, b\right) x_{s p} & =W^{\max } \\
\sum_{i \in I} s_{i} & =S^{\mathrm{min}}
\end{aligned}
$$


Table 3. Input information for academic year 2005-06

\begin{tabular}{ccccccc}
\hline Blocks & $|S|$ & $|C|$ & $|I|$ & $|U|$ & Offered wl & Requested wl \\
\hline 1 \& 2 & 356 & 51 & 79 & 590 & 1504 & 1416 \\
$3 \& 4$ & 328 & 64 & 88 & 279 & 1545 & 1288 \\
5 \& 6 & 302 & 58 & 89 & 151 & 1544 & 1333 \\
\hline
\end{tabular}

$$
\begin{array}{rlrl}
\sum_{s \in S} \sum_{p \in P_{s}, c_{s p}=c(i)} x_{s p} & =U^{\max } & \\
z_{s p i}+s_{i} & \geq C_{i}^{\min } y_{i}, & & \\
E_{s} & \in \mathbb{Z}_{+} & & \forall s \in S \\
I_{c}^{\max }, S_{c} & \in \mathbb{Z}_{+} & & \forall c \in C \\
s_{i} & \in \mathbb{Z}_{+} & & \forall i \in I \\
(x, y, z) \text { satisfy }(1)-(9) . &
\end{array}
$$

\section{The Computational Results}

The computational results for the academic year 2005-2006 are given in this section. This academic year was divided into six blocks. Blocks $1 \& 2$, blocks 3 \& 4 and blocks $5 \& 6$ were scheduled simultaneously.

In all blocks the meetings were on Tuesday morning, Tuesday afternoon, Wednesday morning and Wednesday afternoon. Every morning and afternoon was split into two parts. So both blocks contained eight time slots. More details about the input are given in Table 3. The abbreviation wl stands for workload.

The number of students that requested workload in blocks 1 \& 2 was 356 and the total workload they requested was 1416 . Hence, for each block, an average of two courses of the preference list of 10 courses have to be assigned. The number of students requesting workload decreased during the academic year, because of students who are doing a practical training and students who are finishing their studies. In blocks 5 \& 6 the average requested workload per student is larger than in blocks $3 \& 4$, which is caused by students who still hope to reach the required workload for the academic year by doing some 'extra' courses.

Note that the large number of urgencies in blocks 1 \& 2 can be explained by the fact that first year students are preassigned to courses, because they are not able to make a choice themselves. All first year students have six compulsory courses, which they have to do in the first year. These six courses are set as urgent and only four out of these six courses can be done in each block. The urgencies in blocks 5 \& 6 can be explained by students who are finishing their education and only have some specific courses left to do.

The models introduced in Section 4 are solved by the standard IP solver CPLEX 10.0. The computations are done on an Intel Pentium M, 2.0 GHz 
Table 4. Results for the academic year 2005-06

\begin{tabular}{lrrr}
\hline & Block 1 \& 2 & Block 3 \& 4 & Block 5 \& 6 \\
\hline Runtime CPLEX (s) & 1.38 & 1.53 & 1.67 \\
$U^{\max }$ & 439 & 273 & 134 \\
$S^{\min }$ & 0 & 0 & 0 \\
$W^{\text {max }}$ & 1369 & 1261 & 1300 \\
Average position & 3.30 & 3.64 & 3.87 \\
Bad positions & 8 & 16 & 39 \\
\hline
\end{tabular}

processor with 1.0 GB internal memory. The values of the weighting factors were $W_{p}=10, W_{s}=1$ and $W_{e}=100$. The results for the academic year 2005-06 are given in Table 4

The computation time of CPLEX given in Table 4 is the computation time of CPLEX for solving the fourth subproblem. The computation time of the first three subproblems is even less. What can be concluded is that the computation time of CPLEX is negligible.

In blocks $1 \& 2$ a requested workload of 47 , in blocks $3 \& 4$ a requested workload of 27 and in blocks $5 \& 6$ a requested workload of 33 could not be assigned. Especially in blocks $1 \& 2$ this is caused by the small difference between the requested and offered workload. However, the main causes are preference lists for which it was impossible to assign the requested workload. Some examples of such wrongly chosen preference lists are:

- an empty preference list, because students didn't hand it in on time;

- a preference list with less than ten courses;

- a preference list with not enough different time slots in one of the two blocks;

- a preference list with the same course in multiple positions; there was even a student with the same course ten times on his preference list.

If all students were to hand in a preference list with ten courses and enough different time slots, then in blocks $1 \& 2$ only five students would not be assigned to their requested number of courses, and in blocks $3 \& 4$ and blocks $5 \& 6$ only three students.

Table 4 also shows that in blocks 1 \& 2 only 439 out of 590 urgency requests could be assigned. This can be explained by the fact that in these blocks all courses on the preference list of first year students are set as urgent. Most of those preference lists contain six suitable urgent courses of which at most four are assigned. This means at least two not assigned courses with an urgency for each first year student.

The average position denotes the average of the positions of all courses assigned to a student. On average students request a workload of 4 , which mostly corresponds with four courses. For example, if courses on the positions $1,3,5$ and 7 are assigned, then the average position for this student is 4 . Hence, it can be concluded that students get a lot of courses which are on top of their preference list. 
During the academic year, the average position increases. This can be explained by the fact that in blocks $5 \& 6$ the first year students are allowed to choose four courses from the same course pool as the other students. In blocks 3 \& 4 they were mostly assigned to two out of the six obligatory courses and to two courses chosen by themselves. In blocks $1 \& 2$ they were preassigned to obligatory courses.

If a student is assigned to $i \geq 1$ courses from the 7 th to 10 th position on his preference list he has $i-1$ bad positions. From the number of bad positions it can be concluded that students are assigned to courses at the top of their preference lists. That the number of bad positions increases during the year is explained by the first year students.

\section{Conclusions}

We have formulated, analyzed and solved a real-world timetabling problem that showed up at the department of Industrial Design of the TU Eindhoven. Our successful approach was based on an Integer Linear Programming formulation. The running time that CPLEX needs for solving the resulting instances is negligible. An advantage of an Integer Linear Programming approach is its flexibility. Our experience is that the constraints of the timetabling problem change every academic year and even during the academic year.

The administration and the students of the department of Industrial Design were highly satisfied with the timetables generated by our program. Most students now receive courses that are on top of their preference lists. There still are a few students who are not satisfied, but in most cases this turned out to be solely their own fault: they failed to specify correct preferences in the correct format.

\section{References}

1. Ahuja, R.K., Magnanti, T.L., Orlin, J.B.: Network Flows: Theory, Algorithms and Applications. Prentice-Hall, Englewood Cliffs, NJ (1993)

2. Avella, P., Vasilev, I.: A computational study of a cutting plane algorithm for university course timetabling. Journal of Scheduling 8, 497-514 (2005)

3. Burke, E.K., Kingston, J., Jackson, K., Weare, R.: Automated university timetabling: the state of the art. The Computer Journal 40, 565-571 (1997)

4. Burke, E.K., McCollum, B., McMullan, J.P., Qu, R.: Examination timetabling: A new formulation. In: Proceedings of the 6th International Conference on the Practice and Theory of Automated Timetabling, Brno, pp. 373-375 (August 2006)

5. Burke, E.K., Petrovic, S.: Recent research directions in automated timetabling. European Journal of Operations Research 140, 266-280 (2002)

6. Busam, V.A.: An algorithm for class scheduling with section preference. Communications of the ACM 10, 567-569 (1967)

7. Carter, M.W., Laporte, G.: Recent developments in practical examination timetabling. In: Burke, E.K., Ross, P. (eds.) Practice and Theory of Automated Timetabling. LNCS, vol. 1153, pp. 3-21. Springer, Heidelberg (1996) 
8. Carter, M.W., Laporte, G.: Recent developments in practical course timetabling. In: Burke, E.K., Carter, M. (eds.) PATAT 1997. LNCS, vol. 1408, pp. 3-19. Springer, Heidelberg (1998)

9. Cheng, E., Kruk, S., Lipman, M.: Flow formulations for the student scheduling problem. In: Burke, E.K., De Causmaecker, P. (eds.) PATAT 2002. LNCS, vol. 2740, pp. 299-309. Springer, Heidelberg (2003)

10. Daskalaki, S., Birbas, T.: Efficient solutions for a university timetabling problem through integer programming. European Journal of Operations Research 160, 106$120(2005)$

11. de Werra, D.: An introduction to timetabling. European Journal of Operations Research 19, 151-162 (1985)

12. Dimopoulou, M., Miliotis, P.: Implementation of a university course and examination timetabling system. European Journal of Operations Research 130, 202-213 (2001)

13. Even, S., Itai, A., Shamir, A.: On the complexity of timetable and multicommodity flow problems. SIAM Journal of Computing 5, 691-703 (1976)

14. Feldman, R., Golumbic, M.C.: Constraint satisfiability algorithms for interactive student scheduling. In: IJCAI 1989. Proceedings of the 11th International Joint Conference on Artificial Intelligence, pp. 1010-1016 (1989)

15. Garey, M.R., Johnson, D.S.: Computers and Intractability - a Guide to NPCompleteness. Freeman, San Francisco (1979)

16. Laporte, G., Desrochers, S.: The problem of assigning students to course sections in a large engineering school. Computational Operations Research 13, 387-394 (1986)

17. McCollum, B.: A perspective on bridging the gap in university timetabling. In: Burke, E.K., Rudová, H. (eds.) PATAT 2006. LNCS, vol. 3867, pp. 3-23. Springer, Heidelberg (2007)

18. Miyaji, I., Ohno, K., Mine, H.: Solution method for partitioning students into groups. European Journal of Operations Research 33, 82-90 (1981)

19. Petrovic, S., Burke, E.K.: University timetabling. In: Handbook of Scheduling: Algorithms, Models, and Performance Analysis, ch. 45, CRC Press, Boca Raton, FL (2004)

20. Sabin, G.C.W., Winter, G.K.: The impact of automated timetabling on universities - a case study. Journal of Operations Research Society 37, 689-693 (1986)

21. Schaerf, A.: A survey of automated timetabling. Artificial Intelligence Review 13, 87-127 (1999)

22. Schmidt, G., Ströhlein, T.: Timetable construction - an annotated bibliography. The Computer Journal 23, 307-316 (1980)

23. Tripathy, A.: Computerised decision aid for timetabling - a case analysis. Discrete Applied Mathematics 35, 313-323 (1992)

24. Zampieri, A., Schaerf, A.: Modelling and solving the Italian examination timetabling problem using tabu search. In: Proceedings of the 6th International Conference on the Practice and Theory of Automated Timetabling, Brno, pp. 487491 (August 2006)

\section{A Max-Flow Model of Problem Formulation No. 1}

Problem formulation No. 1 can be modeled as a network flow problem. An illustration of the network with its arc capacities can be found in Figure 1. The layered network has a source node connected to the first layer of nodes of which 
each node corresponds to a student. The capacity of the arcs between a student node and the source node is equal to the requested number of courses of the student. For each student and each time slot, a node is defined in the second layer. Each student node has $|T|$ outgoing arcs with capacity one.

The third layer of the graph contains a node for every course. If a course is on the preference list of a student, this course node is connected with the node of its time slot of the student. For example, in Figure 1 course number 1 is given in time slot 1 . Because course 1 is chosen by student 1 and student $|S|$, there are arcs from nodes $t_{11}$ and $t_{|S| 1}$ to course node $C_{1}$. Each course node is connected to the sink with its maximum number of students as its capacity.

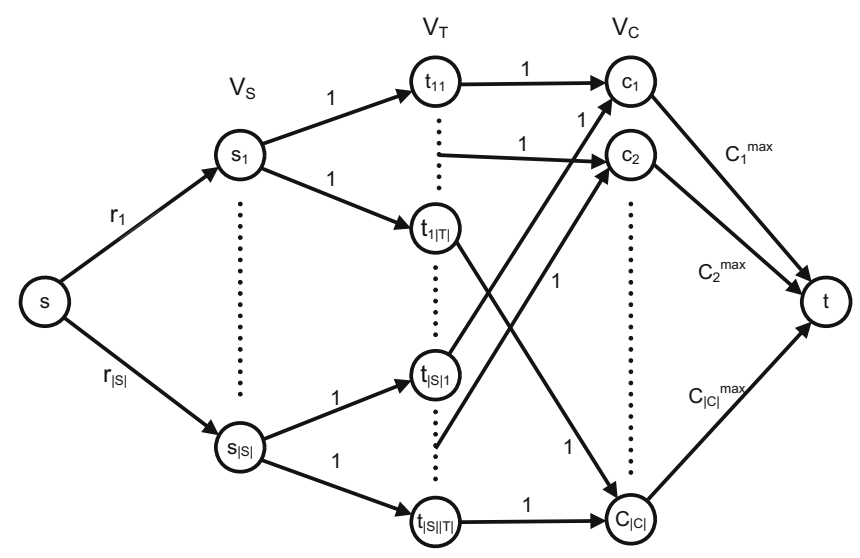

Fig. 1. The network flow model

Note that maximizing the flow through this network maximizes the number of assigned courses and does not deal with the positions of the courses on the preference lists.

\section{B Some NP-Hardness Results}

The timetabling problem defined in Section 3.2 is an NP-hard problem. We prove this by identifying two independent NP-hard subproblems. Both subproblems result from adding one additional constraint to the problem formulation No. 1 .

In the first subproblem, the additional constraint are lower bounds on the number of students in the courses. There are no time slots, there is only one section for each course $c$ with a minimum and a maximum number of participating students. The workload of all courses is one, and only one block has to be scheduled. Formally, problem $P_{\min }$ is defined as follows:

Instance: A set $C$ of courses; for every course $c \in C$ a minimum capacity $C_{c}^{\min }$ and a maximum capacity $C_{c}^{\max }$ of participating students. A set $S$ 
of students; for every student $s \in S$ a preference list of some courses in $C$, and a number $r_{s}$ of requested courses.

Question: Does there exist an assignment such that (i) every student $s$ gets exactly $r_{s}$ courses from its preference list, and such that (ii) for every course $c$ the number of assigned students is either zero (if the course does not take place) or falls between the bounds $C_{c}^{\min }$ and $C_{c}^{\max }$ ?

Theorem 1. Problem $P_{\min }$ is NP-hard.

Proof. The proof is done by reduction from the exact cover by 3-sets problem: Given a ground set $X=\left\{x_{1}, \ldots, x_{n}\right\}$ and a set $T=\left\{t_{1}, \ldots, t_{m}\right\}$ of 3 -element subsets of $X$, can one select $T^{\prime} \subseteq T$ such that every element of $X$ occurs in exactly one member of $T^{\prime}$ ?

From an instance of the exact cover by 3 -sets problem, we construct a corresponding instance of problem $P_{\min }$ with $n$ students $x_{1}, \ldots, x_{n}$ and with $m$ courses $t_{1}, \ldots, t_{m}$. Every student $s$ has a demand of one course $\left(r_{s}=1\right)$, and every course $c$ has minimum and maximum capacity three $\left(C_{c}^{\min }=C_{c}^{\max }=3\right)$.

Assume $X$ possesses an exact cover $T^{\prime}$. Assign student $x_{s}$ to course $t_{c}$ if and only if $x_{s} \in t_{c}$ and $t_{c} \in T^{\prime}$. Since $T^{\prime}$ is an exact cover of $X$, every student $x_{s}$ will be assigned to exactly one course $t_{c}$. The course $t_{c}$ is assigned to three students if it is in $T^{\prime}$, and to zero students if it is not in $T^{\prime}$. This shows that the constructed instance of $P_{\min }$ is a yes-instance. The converse statement can be seen in a similar way.

In the second subproblem, we take problem formulation No. 1 and additionally allow courses with a workload of 2 . We consider a situation with only one section for each course $c$, only a single block, and without any time slots. (And there is no minimum capacity of courses.) Problem $P_{w l}$ is defined as follows:

Instance: A set $C$ of courses; for every course $c \in C$ a workload $w l_{c} \in$ $\{1,2\}$ and a maximum capacity $C_{c}^{\max }$ of participating students. A set $S$ of students; for every student $s \in S$ a preference list of some courses in $C$, and a desired workload $r_{s}$.

Question: Does there exist an assignment such that (i) every student $s$ gets courses with a total workload $r_{s}$ from $P_{s}$, and such that (ii) for every course $c$ the number of assigned students is at most $C_{c}^{\max }$ ?

Theorem 2. Problem $P_{w l}$ is NP-hard.

Proof. The proof is done by reduction from the 3-SAT variant where every variable occurs exactly twice in negated and exactly twice in unnegated form. Consider an arbitrary instance of this 3-SAT variant:

- For every variable $x_{i}$, we introduce two corresponding students $s t\left(x_{i}\right)$ and $s t\left(\overline{x_{i}}\right)$ which both request a workload of two.

- For every variable $x_{i}$, we also introduce a corresponding variable-course $C\left(x_{i}\right)$ which has a workload of two and a capacity of one. $C\left(x_{i}\right)$ is in the preference list of $s t\left(x_{i}\right)$ and $s t\left(\overline{x_{i}}\right)$. 
- For every clause $c_{j}$, we introduce a clause-course $C\left(c_{j}\right)$ with a workload of one and a capacity of two. Clause-course $C\left(c_{j}\right)$ is in the preference list of a student $s t\left(x_{i}\right)$ (respectively $s t\left(\overline{x_{i}}\right)$ ) if and only if $x_{i}$ (respectively $\overline{x_{i}}$ ) occurs as a literal in clause $c_{j}$.

Note that in any feasible assignment, student $s t\left(x_{i}\right)$ (respectively student $s t\left(\overline{x_{i}}\right)$ ) will either do course $C\left(x_{i}\right)$ or the two courses $C\left(c_{j_{1}}\right)$ and $C\left(c_{j_{2}}\right)$ for which literal $x_{i}$ (respectively literal $\overline{x_{i}}$ ) occurs in clauses $c_{j 1}$ and $c_{j 2}$.

Assume that the 3-SAT instance is a yes-instance, and consider a corresponding satisfying truth-assignment. If $x_{i}$ is set to TRUE, then we assign student $s t\left(x_{i}\right)$ to the variable-course $C\left(x_{i}\right)$, and student $s t\left(\bar{x}_{i}\right)$ to the two clause-courses that correspond to the clauses containing $\bar{x}_{i}$. If $x_{i}$ is set to FALSE, we assign $s t\left(x_{i}\right)$ to the clause-courses that correspond to the clauses containing $x_{i}$, and student $s t\left(\overline{x_{i}}\right)$ to $C\left(x_{i}\right)$. Then each student receives his requested workload, and every course $C\left(x_{i}\right)$ gets only a single student. Since every clause has at most two FALSE literals, the corresponding clause-course will get at most two students. So every yes-instance of the 3-SAT problem leads to a yes-instance of the timetabling problem.

Now assume that the constructed instance of problem $P_{w l}$ is a yes-instance. Then every student $s t\left(x_{i}\right)$ receives a workload of 2 , which implies that the student must either be assigned to one course $C\left(x_{i}\right)$, or to two clause-courses $C\left(c_{j 1}\right)$ and $C\left(c_{j 2}\right)$. If student $s t\left(x_{i}\right)$ is assigned to the variable-course $C\left(x_{i}\right)$, we set $x_{i}$ to TRUE. If student $x_{i}$ is assigned to some clause-courses, then we set $x_{i}$ to FALSE. Since each clause-course $C\left(c_{j}\right)$ is assigned to at most two students, every clause contains at most two FALSE literals. Hence, every yes-instance of $P_{w l}$ corresponds to a yes-instance of 3-SAT. 